\title{
Numerical Simulation and Analysis of Non-cavitation noise Line-spectrum Frequency of Underwater Counter-rotation Propeller
}

\author{
ZENG Sai ${ }^{1,2,}$, DU Xuanmin ${ }^{1,2,}$ \\ ${ }^{1}$ Science and Technology on Underwater Acoustic Antagonizing Laboratory, Shanghai, 201108 \\ China. \\ ${ }^{2}$ Shanghai Marine electronic equipment research institute, Shanghai, 201108, China. \\ asharemezeng@hotmail.com
}

Keywords: acoustic analogy equation; counter-rotation propeller; non-cavitation line-spectrum frequency; RNG $k-\varepsilon$ turbulent model; numerical simulation

\begin{abstract}
This paper analyzes the mechanism of non-cavitation tone noise line-spectrum frequency of counter-rotation propeller. The mechanism is interference effect and circumferential harmonics field effect. According to the generalized acoustic analogy equation, the lift and drag which are caused by the mechanism are the source of noise, the far field sound pressure expression is presented. The line-spectrum frequency and the directionality of sound pressure have been analyzed. The results show: the predication line-spectrum frequency could be written as $f=s A P F+m B P F_{1}+n B P F_{2}$; sound pressure radiation is "8"-shaped distribution and the Bessel function's peak condition is a necessary condition for line-spectrum. The counter-rotation propeller's non-cavitation condition is numerically simulated using $R N G k-\varepsilon$ turbulent model、 sliding mesh model and $k \mathrm{FW}-\mathrm{H}$ equation. The results shows that $R N G k-\varepsilon$ Turbulent model has the better accuracy than Realizable $k-\varepsilon$ turbulent model, and the counter-rotation propeller non-cavitation line-spectrum frequency and the directionality of sound pressure are got by this method which agree well with the result of Academic prediction.
\end{abstract}

\section{Introduction}

Sound generated by a propeller is critical in underwater detection, and it is related to the survivability of the vessels especially for military purposes. As the propeller rotates, it is subjected to unsteady force, which leads to discrete tonal noise, and cavitation. Therefore, underwater propeller noise can be classified into cavitating and non-cavitating noise [1]. Counter-rotation propellers have advantage of high propulsive efficiency and torque balance, which have been widely used in underwater high speed vehicles and high speed yacht,ect. In recent years, acoustic stealth indicator is increasingly important for underwater vehicles, propellers have always been the focus of attention which have been treated as an important noise source. Numerous studies focused on the aviation counter rotating rotors. Hanson [2] had built the theory for noise generation of counter-rotation rotors with special emphasis given to the effects of acoustic and aerodynamic interference between the two rotors. Parry [3] used asymptotic techniques the complex expressions for the sound radiated from a counter-rotation rotor were reduced to simple results which do not involve numerical integration or evaluation of Bessel functions but retain the main parametric dependences. Peters [4] had used the method of computational aero-acoustic coupled computational fluid dynamics to analysis the noise field of aviation counter-rotation propellers. With the successful experience of aviation, Zhu [5] had built a prediction method of counter-rotation propeller's tone noise based on a combination of lifting surface theory and acoustic techniques. Wang [6] made vacuole as one part of propeller, using Goldstein equation to predict the line-spectrum frequency of contra-rotating propeller. Based on hybrid numerical method and cavitation tunnel test, Yang [7] had predicted the cavitating inception tonal components and broadband noise of a contra-rotating propeller in the behind-tail condition.

With the improvement of the level of design, the counter-rotation propeller design 
philosophy has been avoiding cavitation for the widest possible range of operating condition. In the mean time, submarines and torpedoes are usually operated under the deep sea enough to avoid cavitations [1]. So both cavitation and non-cavitation noise are also important. Non-cavitation is normal condition of contra-rotating propeller and the noise is significant for underwater target recognition. This paper will study non-cavitation line-spectrum noise of underwater counter-rotation propeller, theoretical analysis and numerical prediction will be used. Noise analysis is performed using frequency-domain acoustic analogy which is deformed by generalized acoustic analogy equation. Noise prediction is performed using Ffowcs Williams-Hawkings equation. Through these studies, the dominant noise source of underwater counter-rotation propeller is analyzed, which will provide a reference for underwater target recognition.

\section{Theoretical and analysis of non-cavitation tonal noise of underwater counter-rotation propeller}

\section{Deformed Generalized acoustic analogy equation}

According to generalized acoustic analogy equation [8], the noise filed of propeller could be described as monopole、 dipole and quadrupole:

$$
\rho^{\prime}=-\frac{1}{c_{0}^{2}} \int_{-T}^{T} \int_{v(\tau)} \frac{\partial^{2} G}{\partial y_{i} \partial y_{j}} T_{i j} d y d \tau+\frac{1}{c_{0}^{2}} \int_{-T}^{T} \int_{s(\tau)} \frac{\partial G}{\partial y_{i}} f_{i} d S(y) d \tau+\frac{1}{c_{0}^{2}} \int_{-T}^{T} \int_{s(\tau)} \rho_{0} v_{n} \frac{\partial G}{\partial \tau} d S(y) d \tau
$$

Here, $\mathrm{dS}(\mathrm{y})=\mathrm{d} \gamma_{o} \mathrm{dr}_{o}$; $\mathrm{S}$ is the body surface. $\mathrm{G}$ is green function. The meaning of negative is that $v_{n}$, $f_{i}$ take the vertical of out surface to be positive direction. Considering that quadrupole and monopole have low radiation efficiency at low mach number and non-cavitation condition, we take the dipole as the main radiation source.

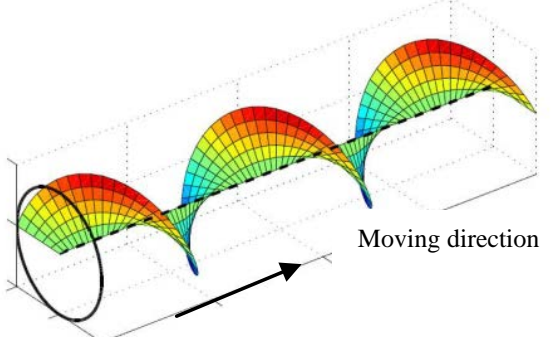

Figure1. Schematic of blade's helical track

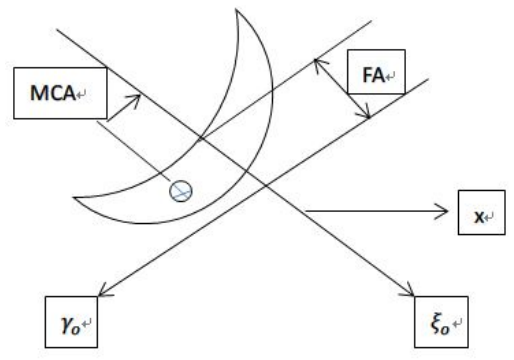

Figure2. Schematic of blade's tangent plane helical coordinate system

The blade's trajectory is helical track which is shown in Fig.2. In order to link the force source of blade surface and far-field sound pressure, three sets of coordinates have been built: geodetic coordinate system $(x, r, \phi)$ 、moving coordinate system $\left(x_{1}, r, \phi\right)$ and helical coordinate system ( $\gamma, r$, $\xi)$. The distance between receiver point and source point $\left(\gamma_{0}, r_{0}, \xi_{0}\right)$ could be written as follow with trylor equation:

$$
R \square r-\frac{\Omega r_{0}}{U_{0}} \xi_{0} \cos \theta+\frac{v}{U_{0}} \gamma_{0} \cos \theta-r_{0} \sin \theta \cos \left(\varphi+\frac{\nu \xi_{0}}{U_{0} r_{0}}+\frac{\Omega \gamma_{0}}{U_{0}}\right)
$$

In the far-field, $1 / R$ could be instead by $1 / r$, as show in Fig. 3.

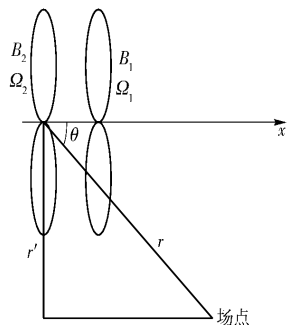

Figure3. Schematic of viewport in field

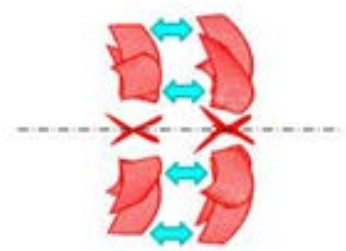
Figure4. Schematic of interference effect and circumferential harmonics field effect Taking Fourier transform [9] into consideration, (1) could be given as follows: 


$$
c_{0}^{2} \rho^{\prime}(x, t)=\iiint \frac{1}{8 \pi^{2} U_{0} R} \int \psi\left(\frac{w^{\prime}}{U_{0}}, \xi_{0}, r_{0}\right) \cdot \exp \left\{i\left[\frac{w^{\prime}+w}{c_{0}} R-\left(w^{\prime}+w_{0}\right) t-\frac{w^{\prime} \gamma_{0}}{U_{0}}\right]\right\} d w^{\prime} d \gamma_{0} d \xi_{0} d r_{0}
$$

Far-field sound pressure by interference effect and circumferential harmonics field effect

The line-spectrum of non-cavitation could be generated by the following eight aspects factors: interaction between non-uniform flow and leading edge of front propeller; the induced unsteady force which cause by large-scale vortex near the blade surface; interaction between after propeller and second unsteady flow of forward blade surface; interaction between front propeller and unsteady pressure field which caused by after propeller's suction; interaction between after propeller's leading edge and front propeller's tip vortex; interaction between after propeller's leading edge and front propeller's trailing edge vortex; interaction between after propeller and boundary layer flow; interaction between after propeller and front propeller's hub wake vortex. The eight aspects factors could be divided into two vocal mechanisms, that's interference effect and circumferential harmonics field effect. The both effects could be described in Fig.4.

Interference effect can be subdivided into three parts: viscous flow field effect; potential flow field effect of trailing edge; boundary layer flow effect. The unsteady force could be used to describe the interference effect. The interference frequency of after propeller which caused by forward propeller is given as follows:

$$
w_{k}=k B_{1}\left(\Omega_{1}+\Omega_{2}\right), \quad(k=0, \pm 1, \cdots)
$$

Taking $\boldsymbol{e}^{\text {iwt }}$ as time factor, the force of after propeller's first blade is shown as follows:

$$
\Delta p_{1}\left(\gamma_{0}, \xi_{0}, r_{0}, \tau\right)=\Delta p_{k}\left(\gamma_{0}, \xi_{0}, r_{0}\right) \cdot \exp \left[i k B_{1}\left(\Omega_{1}+\Omega_{2}\right) \tau\right]
$$

Considering the deformation of special functions[10] and above equations, the far-field sound pressure of after propeller caused by front propeller is shown as follows:

$$
\begin{aligned}
& p_{21}^{\prime}=\frac{i \rho_{0} C_{0}^{2} B_{2} \sin \theta}{8 \pi\left(r_{s} / D\right)\left(1-M a_{x} \cos \theta\right)} \cdot \sum_{n=-\infty}^{\infty} \sum_{k=-\infty}^{\infty} \exp \left\{i\left[\left(m B_{2}-k B_{1}\right)\left(\varphi-\frac{\pi}{2}\right)+\frac{k B_{1} \Omega_{1}+m B_{2} \Omega_{2}}{1-M a_{x} \cos \theta}\left(\frac{r_{s}}{C_{0}}-t\right)\right]\right\} \\
& \cdot \int_{\text {root }}^{t i p} M a_{r}^{2} \exp \left[i\left(\varphi_{r}+\varphi_{s}\right)\right] J_{m B_{2}-k B_{1}}\left[\frac{k B_{1} M_{t 1}+m B_{2} M_{t 2}}{\left(1-M a_{x} \cos \theta\right)} z_{0} \sin \theta\right] \cdot\left[k_{x} \frac{C_{D k}}{2} \psi_{D k}\left(k_{x}\right)+k_{y} \frac{C_{L k}}{2} \psi_{L k}\left(k_{x}\right)\right] d z_{0}
\end{aligned}
$$

Taking circumferential harmonics field effect into consideration, due to blade's action of cutting water, the flow field around the propeller has obvious circumferential harmonics property, the frequency caused by this is shown as follows:

$$
w_{k}=k_{2} w_{2} \Omega_{2},\left(k_{2}, w_{2}=0, \pm 1, \cdots\right)
$$

where, $w_{2}$ is circumferential harmonics order, $k_{2}$ is frequency order. The same method is used to get the far-field sound pressure caused by circumferential harmonics field effect:

$$
\begin{aligned}
& p_{22}^{\prime}=\frac{i \rho_{0} c_{0}^{2} B_{2} \sin \theta}{8 \pi\left(r_{s} / D\right)\left(1-M a_{x} \cos \theta\right)} \cdot \sum_{n=-\infty}^{\infty} \sum_{k_{2}=-\infty}^{\infty} \exp \left\{i\left[\left(k_{2} w_{2}-n B_{2}\right)\left(\varphi-\frac{\pi}{2}\right)+\frac{n B_{2} \Omega_{2}}{1-M a_{x} \cos \theta}\left(\frac{r_{s}}{C_{0}}-t\right)\right]\right\} \\
& \cdot \int_{\text {root }}^{\text {tip }} M a_{r}^{2} \exp \left[i\left(\varphi_{r w}+\varphi_{s w}\right)\right] J_{n B_{2}-k_{2} B_{2}}\left[\frac{n B_{2} M_{t 2}}{\left(1-M a_{x} \cos \theta\right)} z_{0} \sin \theta\right] \cdot\left[k_{x_{w}} \frac{C_{D k_{2}}}{2} \psi_{D k_{2}}\left(k_{x_{w}}\right)+k_{y_{w}} \frac{C_{L k_{2}}}{2} \psi_{L k_{2}}\left(k_{x_{w}}\right)\right] d z_{0}
\end{aligned}
$$

The sum of equation (6) and (8) is the total sound pressure of after propeller. The total sound pressure of forward could be obtained by the same method. The sum sound pressure of forward and after propeller is the sound pressure of counter-rotation propeller. In equation (6) and (8), the parameters could be given in Ref.[8].where, $B_{1}$ and $B_{2}$, respectively, means the blade number of forward and after propeller; $\mathrm{D}$ denotes the diameter of propellers; $r_{\mathrm{i}}$ denotes the distance between receiver and source; $M C A$ and FA denote the skewed distance and trim distance of blade; $\phi^{(i)}$ denotes the initial phase angle; $w$ means the circumferential harmonics field effect; respectively, $M a_{\mathrm{x}} 、 M a_{\mathrm{r}}$ and $M a_{\mathrm{t}}$ denote the forward Mach number, blade sliced Mach number and blade tip mach number; $n, m$ and $k$ denote harmonic order; $C_{D k}, C_{L k}, C_{D w}$ and $C_{L w}$ denote unsteady lift and drag coefficient; $\Psi$ denotes shape function. 


\section{Analysis of radiation formulae}

The radiation formulae contain the characteristics of non-cavitation line-spectrum and directionality of sound pressure. The time entries in the formulae contain the characteristic of line-spectrum frequency, four kind of frequency could be found from radiation formulae, respectively, APF denotes axis frequency, $\mathrm{BPF}_{1}$ and $\mathrm{BPF}_{2}$ denote the forward and after blade frequency, $m B P F_{1}+n B P F_{2}$ is the interaction frequency. The line-spectrum frequency of counter-rotation propeller could be treated as a combination of these four types frequency. It can be shown as follows: $f=s A P F+m B P F_{1}+n B P F_{2}$. (s,m, $n$ would be integer or non-integer ).

The directionality of sound pressure is determined by Bessel Function in the radiation formulae. Discussion will be given about the directionality of after propeller's far-field sound pressure which caused by interference effect. The sound pressure direction function could be defined as follows:

$$
D_{\text {inter }}\left(\theta_{2}\right)=\frac{\left|\int_{z_{02 \text { root }}}^{z_{02 \text { tip }}} J_{N}\left[\frac{n_{2} B_{2} M a_{t 2}+n_{1} B_{1} M a_{t 1}}{1-M a_{x 2} \cos \theta_{2}} \mathrm{z}_{02} \sin \theta_{2}\right]\left(k_{y}^{(2)}\right) d z_{02}\right|}{\max \left|\int_{z_{02 \text { root }}}^{z_{02 \text { rip }}} J_{N}\left[\frac{n_{2} B_{2} M a_{t 2}+n_{1} B_{1} M a_{t 1}}{1-M a_{x 2} \cos \theta_{2}} \mathrm{z}_{02} \sin \theta_{2}\right]\left(k_{y}^{(2)}\right) d z_{02}\right|}
$$

Where, $N=n_{2} B_{2}-n_{1} B_{1}$, it represents the order of Bessel function. $\left(n_{1}, n 2\right)$ represents the harmonic number of noise. The dimensionless directional curves of sound pressure about some orders are shown as Fig.5. It can be seen that the sound pressure has no directional when the order is zero. The directional curves have the shape like " 8 " when the order is 1,2 and 3 . The shape becomes more and more sharp with the increasing of order.

The strength of non-cavitation line-spectrum is also determined by Bessel function. When the Bessel function of interference effect and circumferential harmonics field effect take zero value at the same time, there is no line spectrum. When both take peak value, there must have line spectrum. This could serve as a necessary condition for the non-cavitation line spectrum of counter-rotation propeller.

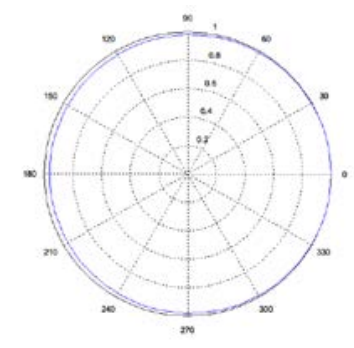

$N=0 \quad\left(\mathrm{n}_{1}=5, \mathrm{n}_{2}=4\right)$

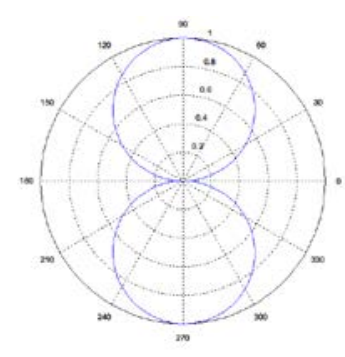

$N=1 \quad\left(\mathrm{n}_{1}=1, \mathrm{n}_{2}=1\right)$

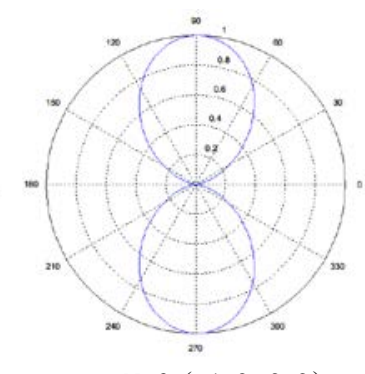

$N=2 （ \mathrm{n} 1=2, \mathrm{n} 2=2)$

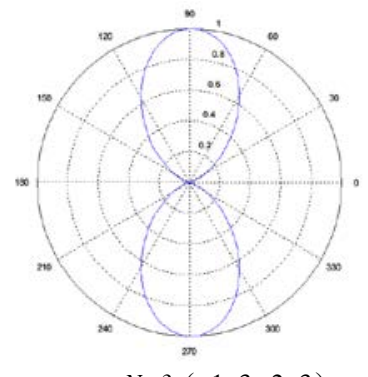

$N=3(\mathrm{n} 1=3, \mathrm{n} 2=3)$

Figure5. Schematic of sound pressure directivity at different order

\section{Numerical simulation and analysis}

\section{Numerical models and meshing}

The Reliable $k-\varepsilon$ turbulent model and RNG $k-\varepsilon$ turbulent model [11] are been used to compute the hydrodynamic performance of model propeller. The simulation data of thrust coefficient $\left(k_{t}\right)$ and torque coefficient $\left(k_{q}\right)$ are compared with open-water test data [12] and then the accuracy turbulence model is chosen to combination with sliding mesh model and FW-H equation to predict the sound field of target propeller. The geometric parameters of model propeller are shown in Table.1. The propeller is shown in Fig.6.

Table1.the geometry parameters of CR propeller

\begin{tabular}{c|c|c}
\hline project & Forward propeller & After propeller \\
\hline Diameter/mm & 305.2 & 299.3 \\
Blade number & 4 & 5 \\
$(P / D)_{0.7 R}$ & 1.291 & 1.287 \\
Expanded Area Ratio & 0.303 & 0.379 \\
Rotation & Left Hand & Right Hand \\
Section Thickness Distribution & NACA 66 & NACA 66 \\
\hline
\end{tabular}




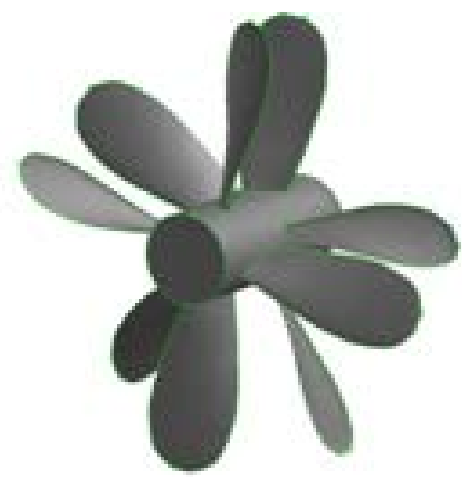

Figure6. Schematic of counter-rotation propeller

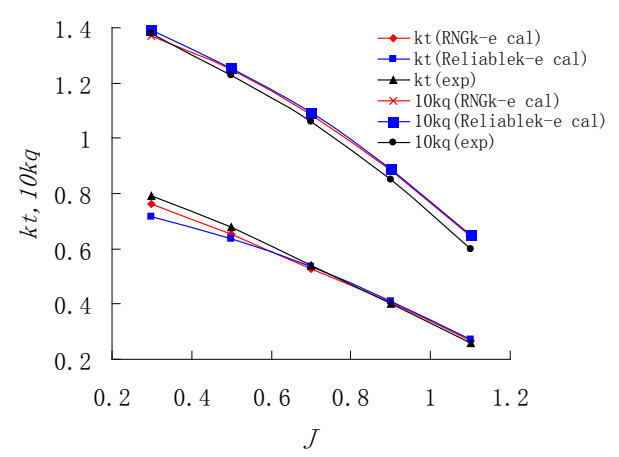

Figure7. The open water performance curves of CR propeller

\section{Results and discussion}

The hybrid mesh method is been used to mesh flow zones. The number of structure and unstructured grid is 1471892 and 2064998. The open water performance curves of model propeller are shown in Fig.7. The definition of counter-rotation propeller's thrust coefficient $\left(k_{t}\right)$ and torque coefficient $\left(k_{q}\right)$ is described in Ref. [13]. The thrust coefficient relative error between calculated value by RNG $k-\varepsilon$ turbulent model and test data is $3.42 \%, 3.70 \%, 2.20 \%, 1.0 \% 、 3.10 \%$. The relative error by Reliable $k$ - $\varepsilon$ turbulent model is $9.10 \%, 6.3 \% 、 1.1 \% 、 2.80 \% 、 4.60 \%$. The torque coefficient relative error between calculated value by RNG $k-\varepsilon$ turbulent model and test data is $0.07 \% 、 1.50 \% 、 0.30 \% 、 3.90 \%$ 、 $7.50 \%$. The relative error by Reliable $k$ - $\varepsilon$ turbulent model is $0.90 \% 、 2.0 \% 、 3.0 \% 、 4.70 \% 、 8.30 \%$. The simulation data of RNG $k-\varepsilon$ turbulent model has better accuracy than Reliable k- $\varepsilon$ turbulent model in hydrodynamic simulation.

The combination of RNG $k-\varepsilon$ turbulent model、sliding mesh and FW-H equation is been chosen to predict the non-cavitation tonal noise of counter-rotation propeller. Firstly, it calculates the flow field under steady state and the result is been saved. Secondary, the result is been treated as initial value to calculate the unsteady flow field, when monitor coefficient come into stability, it means that acoustic field could be simulated. The dynamic stability curves of drag coefficient and thrust coefficient about forward and after propeller in Fig.8 and Fig.9 mean that acoustic field could be simulated. According to Ref [14], step size is been set at 5e-4s.

The density and speed of sound in the undisturbed medium, standard water are $1026 \mathrm{~kg} / \mathrm{m}^{3}$ and $1500 \mathrm{~m} / \mathrm{s}$, respectively. The reference pressure for calculating sound pressure level (SPL) is $1.0 \mathrm{x}$ $10^{-6} \mathrm{~Pa}$. The noise calculations are performed in various receiver positions. The receiver positions are given in terms of the angle $\theta$ and the distance $d$. The receiver P1-P4 and P7-P18 are located at the distance 1.1415 times the forward propeller radius, $\mathrm{R}_{1}$ in the direction of $\theta=180^{\circ}, \theta=270^{\circ}, \theta=0^{\circ}, \theta=90^{\circ}$ and $\theta=30^{\circ}, \theta=45^{\circ}, \theta=60^{\circ}, \theta=120^{\circ}, \theta=135^{\circ}, \theta=150^{\circ}, \theta=210^{\circ}, \theta=225^{\circ}, \theta=240^{\circ}, \theta=3$ $00^{\circ}, \theta=315^{\circ}, \theta=330^{\circ}$ from the propeller shaft axis. The receiver P5 and P6 are located at the distance $\mathrm{R}_{1}$ in the direction of $\theta=270^{\circ}$ from the propeller shaft axis. The receivers' positions are shown in Fig.10. The counter-rotation propeller is assumed to be operated at $1200 \mathrm{rpm}$ with forward velocity of $6.7 \mathrm{~m} / \mathrm{s}$.

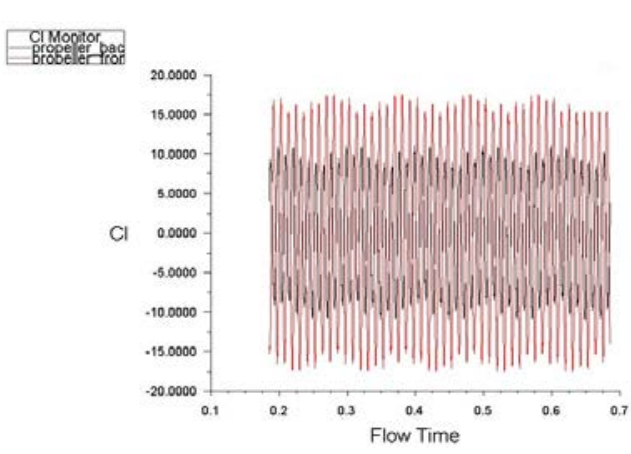

Figure8. The lift coefficient curves of CR propeller

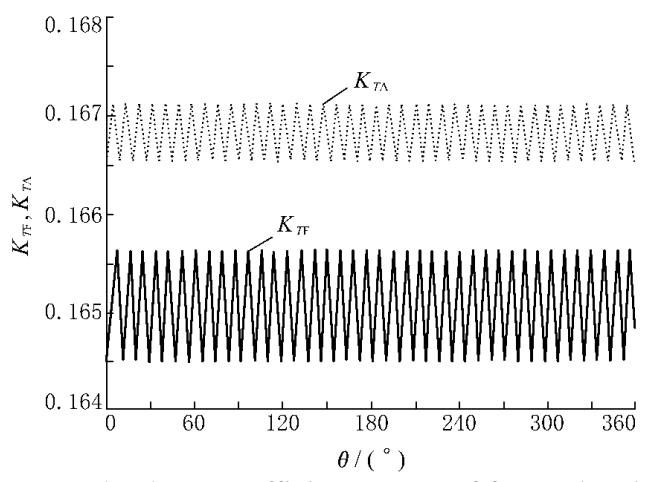

Figure9. The thrust coefficient curves of forward and after propeller in one periodic time 


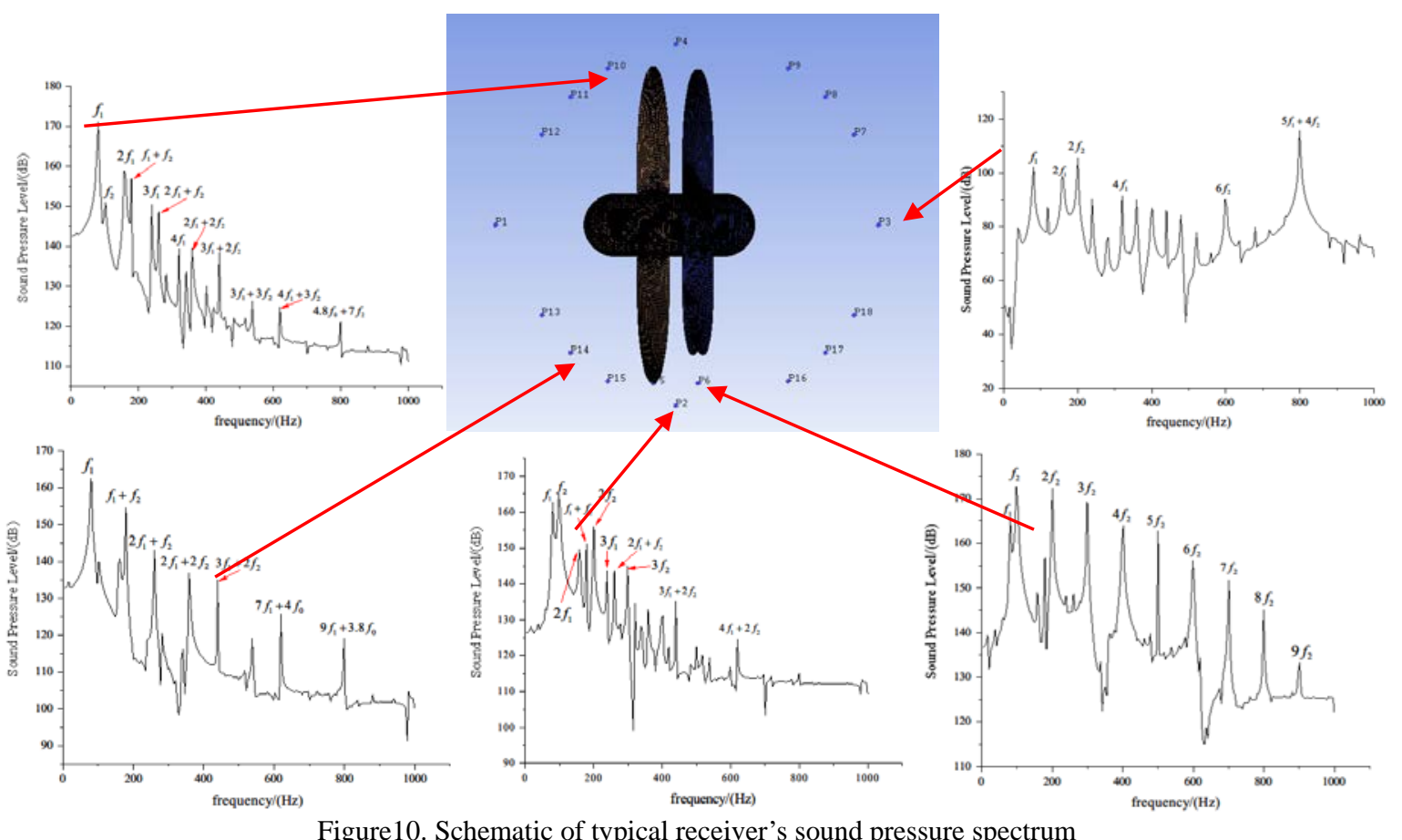

The sound pressure level spectra calculated at various positions are shown in Fig.10. Taking the sound pressure level (SPL) of axial direction receiver P3 into consideration, the frequencies of line spectrum almost are blade passing frequency (BPF)and its' harmonic, the peak line spectrum frequency is $5 f_{1}+4 f_{2}\left(f_{1}=\mathrm{BPF}_{1}, f_{2}=\mathrm{BPF}_{2}\right)$. It means that interference effect is strong in axial direction. The line spectrum frequencies are APF and harmonic of APF in the SPL spectrum of radial direction receiver P2 and P6. It means that circumferential harmonics field effect is strong in radial direction. Considering the SPL spectrum of receiver P10 and P14 which positions are between axial and radial direction, the line spectrum frequencies are combination of BPF. It means that both interference and circumferential harmonics field have effect in this zone.

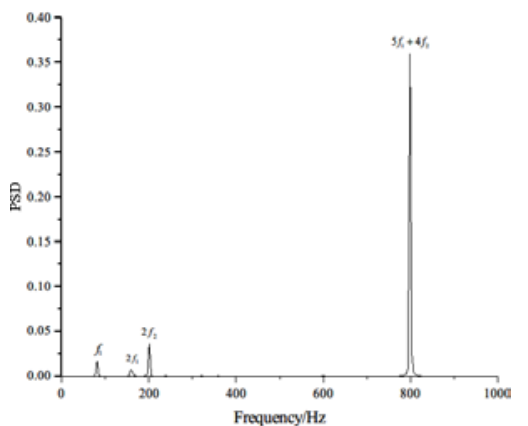

Figure11.Power spectrum of receiver 3

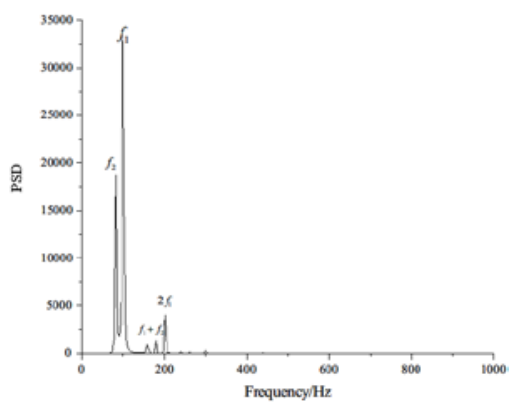

Figure13.Power spectrum of receiver 2

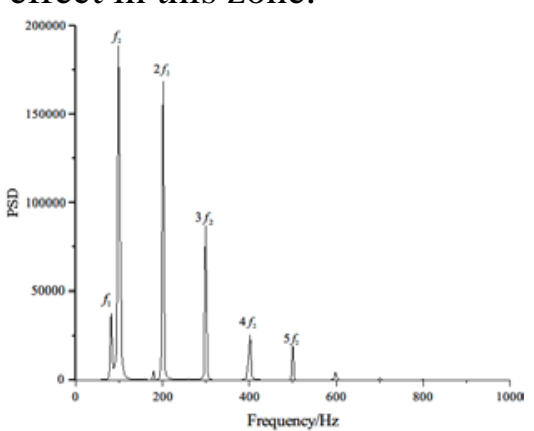

Figure12.Power spectrum of receiver 6

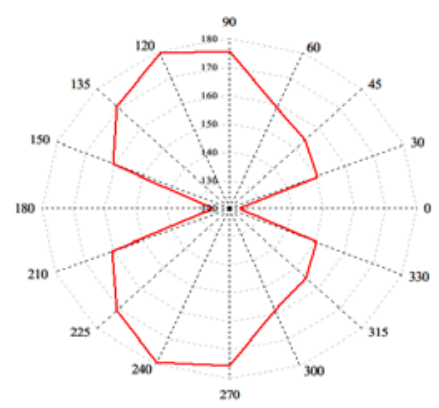

Figure14.SPL curve of receiver

The power spectra calculated at various positions are shown in Fig.11-13. The peak frequencies of power spectra are consistent with SPL spectra in Fig.10. The power spectra are gradually decreased with the increasing order of BPF. The peak power spectra correspond to the first order BPF. Connecting the total SPL of typical receivers into a curve, it is shown in Fig.14. It could be seen that the curve is a simple 8 shaped. 


\section{Conclusion}

The non-cavitation noise generated by a counter-rotation propeller is analyzed numerically and theoretically in this study. This can provide a reference for identification of underwater target. Some conclusions are given as follow:

(1). the non-cavitating vocal mechanisms of counter-rotation are interference effect and circumferential harmonics flow field effect. This makes the line spectrum of noise to be rich.

(2). the line-spectrum frequency of counter-rotation propeller could be written as follows: $f=s A P F+m B P F_{1}+n B P F_{2}$. (s, $m, n$ can be integer or non-integer ).

(3). $u$ RANS method can achieve precision compliant with engineering applications. The simulation data of RNG $k-\varepsilon$ turbulent model has better accuracy than Reliable k- $\varepsilon$ turbulent model in hydrodynamic simulation.

(4). the non-cavitation radiated sound pressure of underwater counter-rotation propeller has directionality. The total sound pressure of radial direction is stronger than axial direction.

(5). The hybrid method of combining uRANS method with FW-H equation can predict the non-cavitation line spectrum noise of underwater counter-rotation propeller. The simulation date agrees well with theory in predicting line spectra.

\section{References}

[1] D. Ross, Mechanics of Underwater Noise, Pergamon Press, Oxford, 1976.

[2] Hanson, D. B. Noise of counter-rotation propellers. J. Aircraft. Vol.22 (7) (1985) p609-617.

[3] Parry, A. B. Theoretical prediction of counter-rotation propeller noise. Ph.D. Thesis, University of Leeds (1988).

[4] Peters A. Assessment of propfan propulsion systems for reduced environmental impact. Master thesis, USA: Massachusetts Institute of Technology, 2010.

[5] ZHU Xiqing, WU Wusheng. Prediction of line-spectrum noise induced by high speed vehicle counter-rotation propellers in water. ACTA ACUSTICA . Vol.23 (2) (1998) 123-134.

[6] WANG Shun-jie, CHENG Yu-sheng, GAO Xin. Prediction and Numerical Simulation of Cavitation Noise Line-spectrum Frequency Induced by Underwater Counter-rotation Propeller. ACTA ARMAMENTAR. Vol.34(3) ( 2013) 311-319.

[7] YANG Qiongfang, WANG Yongsheng, et al. Numerical prediction of cavitation inception radiated noise of contra-rotating propeller with non-uniform inflow. ACTA ACUSTICA, Vol.39(5) (2014)590-606.

[8] SUN Xiaofeng, ZHOU Sheng. Aeroacoustics, Defense Industry Press, Beijin, 1993.

[9] QIAO Weiyang. Aeroengine Aeroacoustics, Beijin Astronautics University Press, Beijin,2010.

[10] LIANG Kunmiao. Methods of Mathematical Physics, Higher Education Press, Beijin, 2011.

[11] WANG Fujun, Computational Fluid dynamics analysis, Tsinghua University Press, 2010.

[12] MILLER. Experimental Determination of Unsteady Forces on Counter-rotating Propellers in Uniform Flow. David Naval Ship Research and Development Center Report SPD-659-01,May 1976.

[13] ZHANG Tao, CHEN Yan-Yong, YANG Chen-Jun. Study on the Prediction of Contra-Rotating Propellers Open-Water Steady Performance Using RANS Formula. SHIP ENGINEERING, Vol.33(5) (2011)23-26.

[14] MA Xukun. Preliminary modeling of line spectrum for radiated noise induced by high -speed counter rotation of underwater vehicle propellers. ACTA ACUSTICA, Vol.27(6) (2002)503-50 